\title{
OPTIMIZATION OF FEEDBACK CONTROL INPUTS FOR POSTURE CONTROL OF A SIX-LEGGED ROBOT
}

\author{
HIROAKI UCHIDA \\ Department of Mechanical Engineering, National Institute of Technology, Kisarazu \\ College, 2-11-1 Kiyomidai-higashi, Kisarazu, Chiba 292-0041, Japan
}

\begin{abstract}
In this study, an optimization method of feedback control inputs for a posture control of a six-legged robot was developed. The authors had proposed a method to control using an optimum servo system as a posture control method of a six-legged robot. As a problem of this method, because the feedback (FB) gain was switched at the time of switching the swing leg, the control inputs becomes discontinuous and there was a problem that the posture variation increases. After that, FB inputs of the thigh link obtained by optimum servo system were optimized. Then, we design a control system that suppressed the posture variation that occurs during swing leg switching. The effectiveness of the proposed control method was confirmed using a 3D model of a six-legged robot.
\end{abstract}

Keywords : Six-legged robot; Posture control; Optimization; Optimum servo control

\section{Introduction}

Multi-legged robots can operate under extreme conditions where it is difficult for humans to work. Walking robots are considered to be active on uneven terrain, and posture control is important for walking. In terms of posture control, there are studies that examined the subject from the perspective of control methods that consider robot dynamics [1][2]. In one such study [2], the authors used a sixlegged robot with a leg mechanism proposed by Hirose et al. [3] to investigate a posture control method. The body height, pitching angle, and rolling angle were controlled by the thigh link during walking with support legs, and a 3D simulation was used to demonstrate the method's effectiveness. As a problem of this method, because the feedback (FB) gain is switched at the time of switching the swing leg, the control inputs becomes discontinuous and there is a problem that the posture variation increases. In this study, we construct an optimal servo system using a mathematical model considering the dynamics of the thigh link proposed by the authors. Moreover, FB inputs of the thigh link obtained by optimum servo system are optimized. Then, we design a control system that reduces the posture variation that occurs during swing leg switching. The effectiveness of the proposed control method is confirmed using a 3D model of a six-legged robot. 


\section{Six-Legged Robot}

Figure 1 shows the six-legged robot used in this study. Each leg of the six-legged robot consists of three joints, a rotating part, a thigh part, and a shank part. Table 1 shows the dimensions of the six-legged robot.

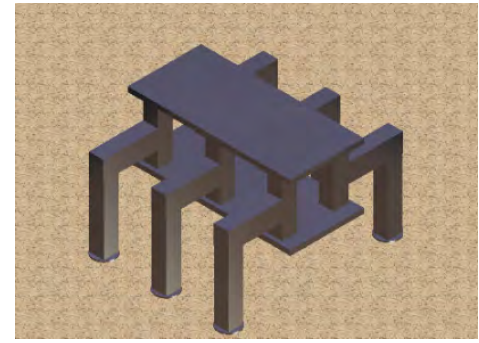

Table 1 Robot dimensions

\begin{tabular}{|c|c|c|c|}
\hline Height & $500 \mathrm{~mm}$ & $\begin{array}{c}\text { Length of } \\
\text { Thigh }\end{array}$ & $168 \mathrm{~mm}$ \\
\hline Length & $666 \mathrm{~mm}$ & $\begin{array}{c}\text { Length of } \\
\text { Shank }\end{array}$ & $312 \mathrm{~mm}$ \\
\hline Width & $710 \mathrm{~mm}$ & $\begin{array}{c}\text { Moment of } \\
\text { Inertia } I_{\mathrm{z}}\end{array}$ & $\begin{array}{c}1.966 \\
\mathrm{kgm}^{2}\end{array}$ \\
\hline Weight & $24.9 \mathrm{~kg}$ & . & \\
\hline
\end{tabular}

Fig. 1 3D CAD model of a 6-legged robot

\section{Walking Planning}

The effectiveness of the proposed control method is verified by the walking pattern of five support legs and one swinging leg. Walking period is 9 sec. Figure 2 shows Leg number and Figure 3 shows walking pattern.

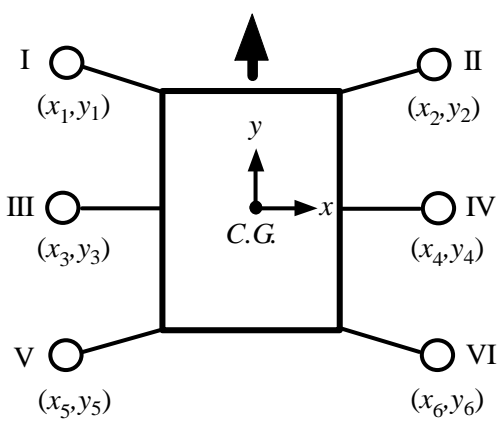

Fig.2 Leg number and position

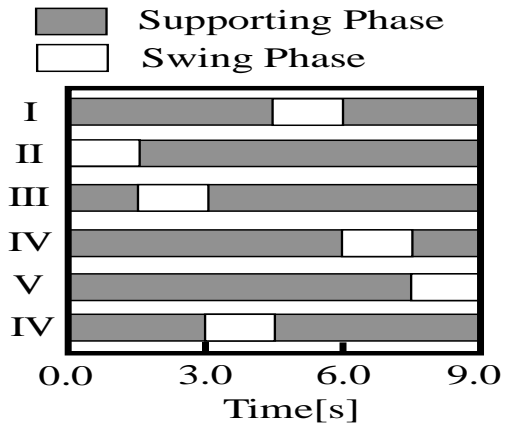

Fig.3 Walking pattern

\section{Posture Control Method [4]}

\subsection{Mathematical Model of the Thigh Link}

The thigh links are driven by the DC motor in this study, and the DC motor is controlled by the current. The equilibrium equation of the torque becomes the next equation for the thigh link shown in Figure 4.

$$
T_{i}=K i_{a}=J \frac{d^{2} \theta_{2 i}}{d t^{2}}+c \frac{d \theta_{2 i}}{d t}+F_{i} l_{t i} \cos \theta_{2 i}
$$




\subsection{Mathematical Model from the Input of the Thigh Link to the Posture of the Body}

The force $F_{i}$ in the perpendicular direction of the supporting leg is given by the following equation:

$$
F_{i}=K_{e} l_{t i}\left\{\sin \theta_{2 i}-\sin \left(\Delta \theta_{2 e q i}\right)\right\}+C_{e} \dot{\theta}_{2 i} l_{t i} \cos \theta_{2 i}
$$

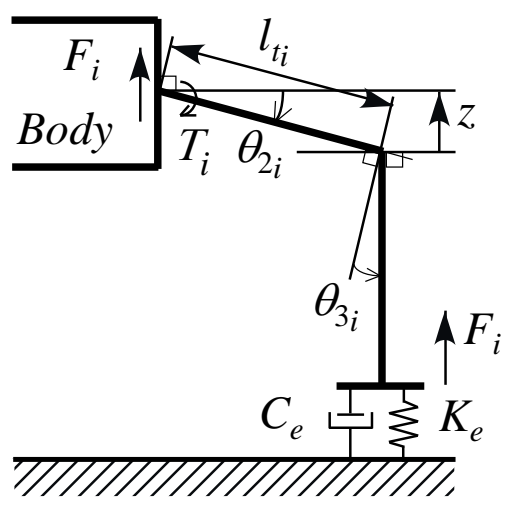

Fig.4 Relationship between the angle of thigh and the force in the perpendicular direction of the supporting leg

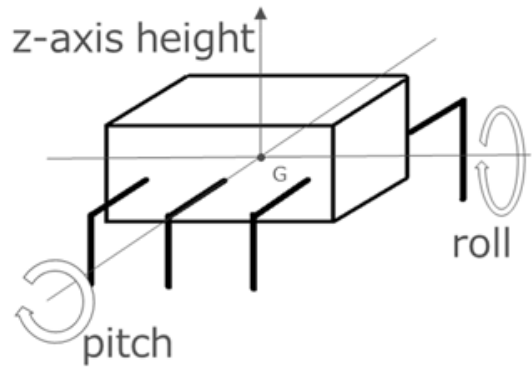

Fig.5 Three parameters of a six-legged robot for posture control

Moreover, the height, and the pitching and rolling angles of the body are controlled by controlling the force in the vertical direction of the support leg. The motion equations of the force and the moment equilibrium in the vertical direction and the pitching and rolling axes in the case of support by six legs are given by Eq. (3) [2][4]. Fig.5 shows the controlling parameters of the body posture.

$$
\left\{\begin{array}{c}
M \ddot{z}=F_{1}+F_{2}+F_{3}+F_{4}+F_{5}+F_{6}-M g \\
I_{p} \ddot{\theta}_{p}=y_{1} F_{1}+y_{2} F_{2}+y_{3} F_{3}+y_{4} F_{4}+y_{5} F_{5}+y_{6} F_{6} \\
I_{r} \ddot{\theta}_{r}=x_{1} F_{1}+x_{2} F_{2}+x_{3} F_{3}+x_{4} F_{4}+x_{5} F_{5}+x_{6} F_{6}
\end{array}\right.
$$

Here, it should be noted that the thigh link range of movement used in this study is $-30^{\circ}$ to $+30^{\circ}, \theta_{2 i}$ is linearized at zero degrees. Substituting Eq. (1) and (2) for Eq.(3), and, by defining the $18^{\text {th }}$ order state value $x=\left[x_{11}, x_{12}, \cdots, x_{61}, x_{62}\right.$, $\left.z, \theta_{p}, \theta_{r}, \dot{z}, \dot{\theta}_{p}, \dot{\theta}_{r}\right]$ which consist of the state values of each thigh link, pitching and rolling angles, the height of the body and their velocity, the following state equation is obtained. In Eq.(4), explanations of $A, B, C, d$ and $f$ are omitted.

$$
\begin{gathered}
\dot{x}(t)=A x(t)+B u(t)+d g+f \\
y(t)=C x(t)
\end{gathered}
$$




\subsection{Posture Control Method}

The servo system that the system shown by Eq. (4) follows to the desired value is designed.

$$
\left\{\begin{array}{c}
\dot{z}(t)=r(t)-C x(t) \\
\dot{x}(t)=A x(t)+B u(t)+d g+f
\end{array}\right.
$$

Equation (5) is described in equation form as follows:

$$
\dot{x}_{g}(t)=A_{g} x_{g}+B_{g} u+d_{g} g+f_{g}+e_{g} r
$$

The control input to the actuator driving the thigh link is obtained to minimize the following equitation.

$$
J=\int_{0}^{\infty}\left(x_{g}(t)^{T} Q x_{g}(t)+u(t)^{T} R u(t)\right) d t
$$

Here, $Q(n \times n)$ and $R(m \times m)$ are the weighting matrix given by the design specification, and $Q \geq 0, R>0$. The control input $u_{b}^{o}$ to minimize Equation (7) is shown as following:

$$
u_{b}^{o}=-R^{-1} B_{g}^{T} P x_{g}
$$

Here, $P(n \times n)$ is the solution of the following Ricatti equation.

$$
P A_{g}+A_{g}^{T} P-P B_{g} R^{-1} B_{g}^{T} P+Q=0
$$

The feature of the proposed control method is that angles of the thigh links controlling the body posture are estimated by the detection of the pitching, rolling and the height of the body. In this study, in order to examine the walking pattern of the five support legs, six sets of FB controller are switched and applied each time the swing leg changes. Therefore, there is a case that the inputs of the support legs greatly change at the moment when the control system switches.

\section{Optimization of Control Input}

In this chapter, we consider a method to further optimize the control inputs obtained by the optimum servo system designed in Chapter 4. An evaluation function is necessary for optimization. Here, we consider the relation between vertical forces generated by the FB inputs obtained in Chapter 4 and the ideal forces in the support legs. We consider the squares of the difference between the ideal force, ideal moments $\left(F^{*}, M_{x}^{*}, M^{*}\right)$ and the actual force, actual moments $\left(F, M_{x}, M_{y}\right)$, and the weight values $\left(\alpha_{F}, \alpha_{x}, \alpha_{y}, \alpha_{\Delta}\right)$ are set. Then, the evaluation function is set as follows:

$$
J=\frac{1}{2} \alpha_{F}\left(F-F^{*}\right)^{2}+\frac{1}{2} \alpha_{x}\left(M_{x}-M_{x}^{*}\right)^{2}+\frac{1}{2} \alpha_{y}\left(M_{y}-M_{y}^{*}\right)^{2}
$$




$$
+\frac{1}{2} \alpha_{\Delta} \sum_{i=1}^{N}\left(f_{i}-\frac{F}{N}\right)^{2}
$$

Equation (10) is an evaluation function. The outputs are the forces in the vertical direction of the support legs. The inputs are constructed by the three components, first one is the body force in the vertical direction and second and third one are the rotational moments in the $x$ - and $y$-axis. The first term on the right side of the Eq. (10) is the error sum of squares with respect to the force in the z-axis direction, and the second term and the third term on the right side are error sums of squares with respect to the moment about the $x$-axis and $y$-axis. The fourth term on the right side is the error sum of squares with respect to the average generating force of all the actuators and the generated force of each actuator. In this study, to minimize the value of $J$, we use "fminsearch" function which is a solver of MATLAB 's Optimization Toolbox. This solver uses the Nelder-Mead algorithm [5], which searches for function minimization without using a derivative function. The ideal force and moments $\left(F^{*}, M^{*}, M^{*}\right)$ expressed by Eq.(10) are defined by following equations.

$$
\begin{gathered}
F^{*}=M_{\text {height }} *\left(z_{\mathrm{r}}-z\right)-C_{\text {height }} * \dot{z}+M \\
M^{*}{ }_{x}=-M_{\text {pitch }} * \theta_{p}-C_{\text {pitch }} * \dot{\theta}_{p} \\
M^{*}{ }_{y}=-M_{\text {roll }} * \theta_{r}-C_{\text {roll }} * \dot{\theta}_{r}
\end{gathered}
$$

$M_{\text {height }}, M_{\text {pitch }}, M_{\text {roll }}, C_{\text {height }}, C_{\text {pitch }}, C_{\text {roll }}$ are the virtual spring constants and the virtual damping coefficients about the position of the $z$-axis, the roll angle and pitch angle. $\mathrm{z}_{\mathrm{r}}$ is the target value of the body in $z$-axis.

Figure 6 shows the block diagram with the optimization part shown in this chapter added to the optimum servo system shown in Chapter 4 . The control input $u$ of the support legs obtained by the optimum servo system is corrected to $u^{*}$ by the optimization method and it is used as the control inputs of the thigh links.

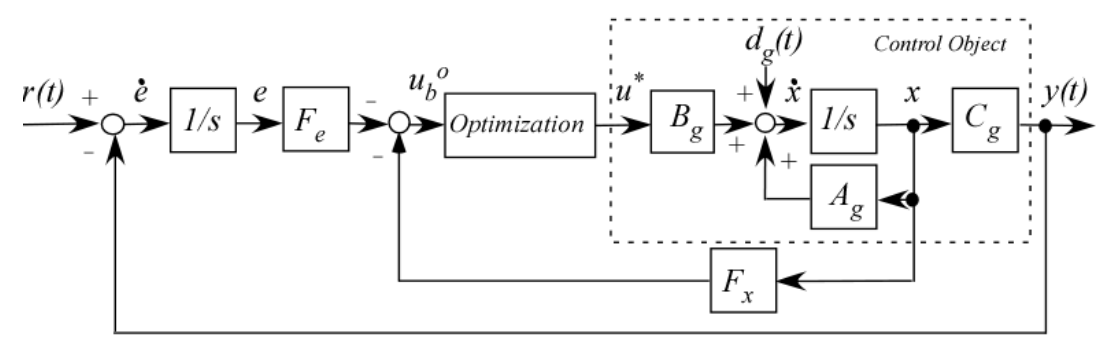

Fig.6 Block diagram of optimal servo with optimization

\section{3D Simulation}

The 3D simulation results are shown in Figure 7. Since the Nelder-Mead method is an iteration method, we performed a simulation with the maximum iteration 
number as 200 in this study. $Q=\operatorname{diag}\left(10^{3}, 10^{3}, 10^{3}, 0, \cdots, 0\right), \quad R=$ $\operatorname{diag}(1,1,1)$ in Eq.(7). Also, $\left(\alpha_{F}, \alpha_{x}, \alpha_{y}, \alpha_{\Delta}\right)$ in Eq.(10) were set to $(1,1,1,1)$. $\left(M_{\text {height }}, M_{\text {pitc }}, M_{\text {roll }}\right)$ are $\left(10^{3}, 10^{3}, 10^{3}\right)$ in Eq.(11), $\left(C_{\text {height }}, C_{\text {pitc }}, C_{\text {roll }}\right)$ are $\left(10^{1}\right.$, $10^{1}, 10^{1}$ ). These parameters were determined by trial and error. Fig.7 (a) is time response of the height of the body, Fig.7 (b) is the pitching angle, Fig.7 (c) is the rolling angle, respectively. In the figure, the red solid line and the green dash-anddotted line show the cases with optimization where iteration number is 200 and 50 , the blue dashed line shows the case without optimization where posture control is performed with the FB input obtained by the optimum servo system shown in Chapter 4, and the black dotted line shows the target trajectory. In Fig. 7 (a) - (c), in the case of controlling the support legs with the FB gain obtained by

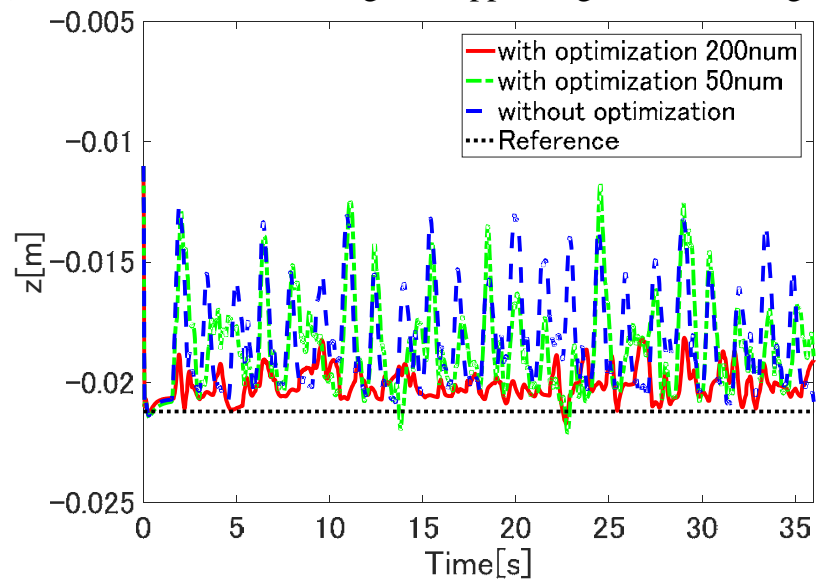

(a) Height of the body

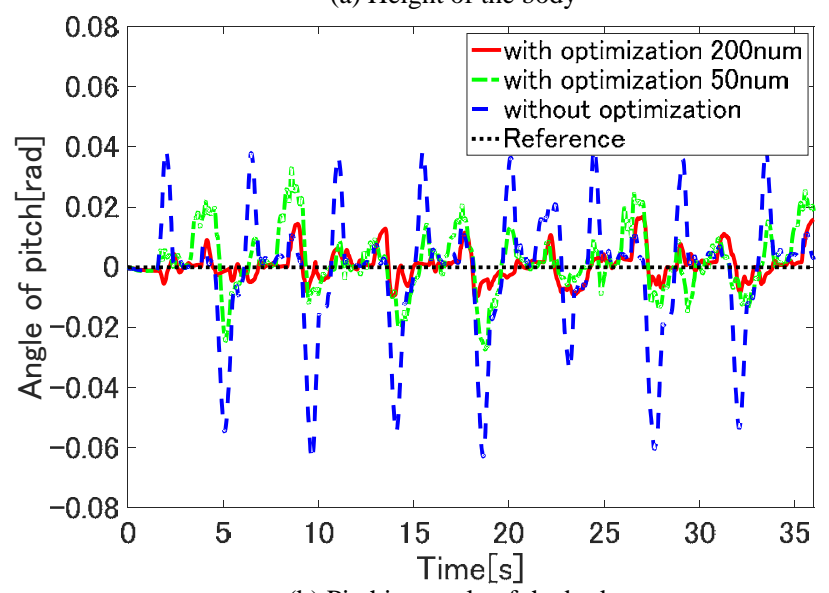

(b) Pitching angle of the body

Fig.7. Simulation results on even terrain 


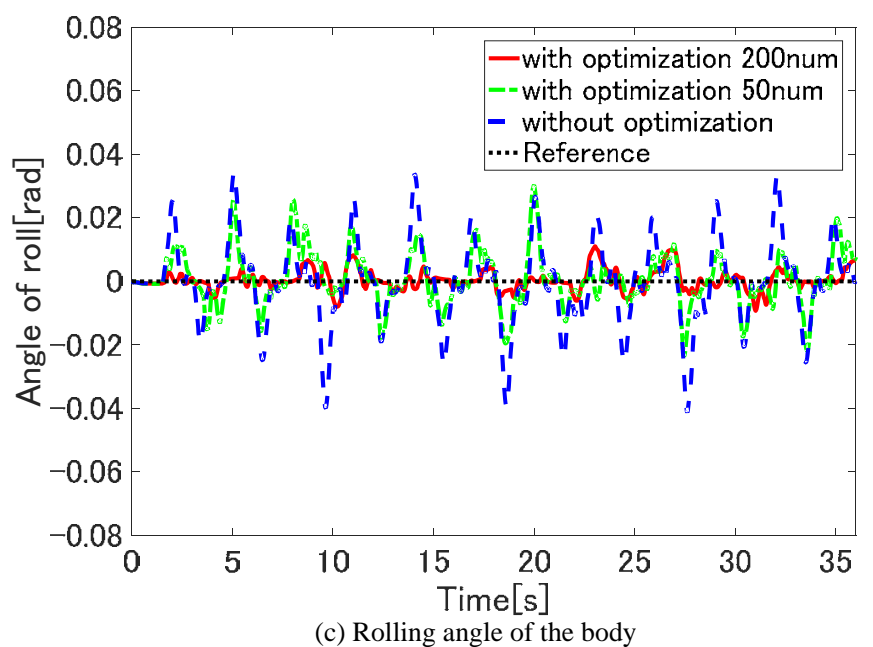

Fig.7. Simulation results on even terrain
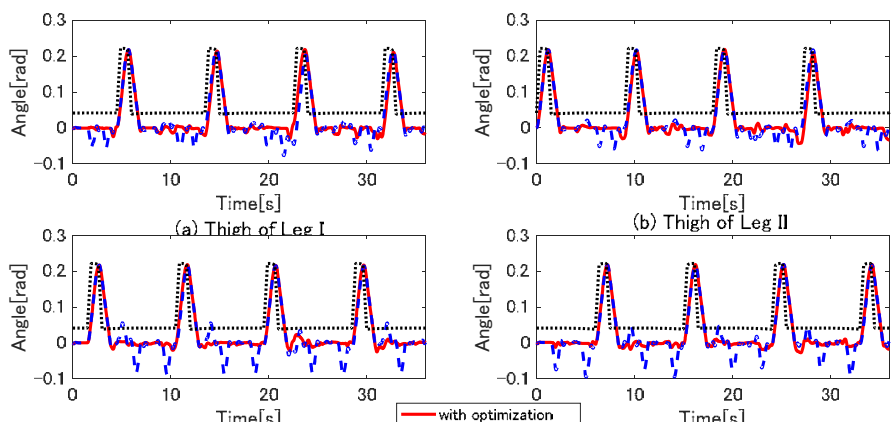

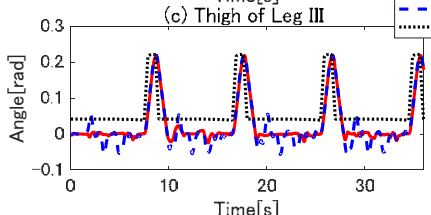

(e) Thigh of Leg $V$ (b) Thigh of Leg II

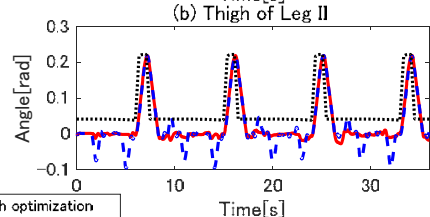

(d) Thigh of Leg IV

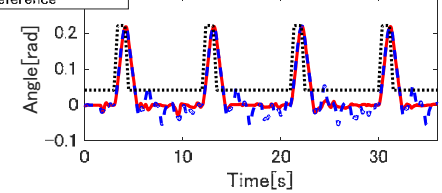

(f) Thigh of Leg VI

Fig.8 Time response of thigh angle

the optimum servo system without optimization, posture variation occurs because the FB control system switches at the time of changing the swing leg. However, when applying the optimization method, it can be seen that large variation occurring at the time of switching the swing legs are reduced in all control amounts of the body height, the pitching angle, and the rolling angle. Especially, with pitching angle and rolling angle, the maximum deflection width is reduced to less than half, and improvement in control performance can be seen. The 
deflection width is suppressed to about half in the pitch angle where iteration number is 50 . We can see the effectiveness of the proposed control method. Figure 8 shows the time response of the thigh angle in the case of the posture control shown in Fig.7. In the case that the optimization is not performed, the thigh angle variation becomes large at the time of switching the swing leg, but the case that the optimization is performed, it can be seen that this large variation is reduced. From these results, it was shown that by applying the optimization method proposed in this study to the posture control of the 6-legged robot, it is possible to reduce the variation of the input due to switching of the control system generated at the time of switching the swing leg.

\section{Conclusion}

In this study, we examined a control method to optimize the control input obtained by the optimum servo system as a posture control method of a six-legged robot and verified the effectiveness of the proposed method by 3D simulation of a sixlegged robot.

1. The design procedure of the optimum servo system as the posture control method of the six-legged robot and the method to optimize the FB control input obtained by the optimum servo system are shown.

2. The effectiveness of the proposed control method was confirmed by 3D simulation using the 3D CAD model of the six-legged robot. Specifically, in the case of walking with five support legs, when the optimization is not performed, a large variation occurs in the height of the body, the pitching angle, and the rolling angle at the time of switching the swing leg. However, when the optimization method is applied, the variation was reduced.

\section{References}

1. Q. Huang, Y. Fukuhara and X. Chen, Posture and Vibration Control Based on Virtual Suspension Model Using Sliding Mode Control for Six-Legged Walking Robot, Jour. of System Design and Dynamics, 1, 2(2007), pp.180191.

2. H. Uchida and K. Nonami, Attitude Control of a Six-legged Robot in Consideration of Actuator Dynamics by Optimal Servo Control System, Climbing \& Walking Robots Towards New Application, I-Tech, 2007, pp 299-312.

3. S. Hirose, and K. Arikawa, Development of quadruped walking robot TITAN-VIII, Proc. of the 1996 IEEE/RSJ International Conference on Intelligent Robots and Systems, vol.1(1996), pp.208-214.

4. H.Uchida, Six-degree-of-freedom Control by Posture Control and Walking Directional Control for Six-legged Robot, Journal of Mechanical Engineering and Automation, 7, 2(2017), pp.30-45.

5. Nelder, John A., R. Mead., A simplex method for function minimization, Computer Journal, 7, 1965, pp.308-313. 